\title{
Consolider la paix dans un contexte de Covid-19 : défis, initiatives et perceptions en République Démocratique du Congo
}

\author{
Yvon Muya et Alain-Parfait Ngulungu*, Université Saint Paul, \\ Université de Kinshasa
}

\section{Résumé}

\begin{abstract}
"Biographie:
Yvon Muya est doctorant à l'école d'études de conflits de l'Université SaintPaul d'Ottawa. Journaliste formé à l'Institut facultaire des Sciences de l'Information et de la Communication de Kinshasa, il analyse les conflits politiques en République Démocratique du Congo depuis près de 20 ans. Sa thèse doctorale traite d'ethno nationalisme et violence. II travaille sur plusieurs sujets: la violence politique, les mouvements citoyens ou encore la justice sociale.
\end{abstract}

Alain-Parfait Ngulungu est doctorant en économie à l'Université de Kinshasa. Titulaire d'une maîtrise en économie durable, il est engagé depuis 2008 dans plusieurs projets mis en œuvre par les organisations internationales en RDC, notamment le PNUD, la Banque mondiale ou Transparency international en Afrique centrale. Ses intérêts de recherche sont variés: la gouvernance climatique, les politiques et législations forestières et climatiques, l'économie politique de la sécurité alimentaire, etc.

\section{Biography:}

Yvon Muya is a doctoral student at the School of Conflict Studies at Saint Paul University in Ottawa. A journalist trained at the Institut facultaire des Sciences de I'Information et de la Communication in Kinshasa, he has been analysing political conflicts in the Democratic Republic of Congo for nearly 20 years. His doctoral thesis deals with ethno nationalism and violence. He works on several subjects: political violence, citizen movements and social justice.

Alain-Parfait Ngulungu is a PhD student in economics at the University of Kinshasa. He holds a master's degree in sustainable economics and has been involved since 2008 in several projects implemented by international organisations in the DRC, notably the UNDP, the World Bank and Transparency International in Central Africa. His research interests are varied: climate governance, forestry and climate policies and legislation, the political economy of food security, etc. 
Cette recherche traite d'effets de la Covid-19 sur la population la plus démunie en République Démocratique du Congo (RDC). Les mesures restrictives prises par les autorités congolaises pendant la première phase de la pandémie pour contenir la crise ont engendré de nouveaux problèmes. Elles ont considérablement affecté les conditions socio-économiques déjà préoccupantes des groupes les plus vulnérables. Avec aussi peu de moyens dont dispose le pays, la volonté du gouvernement d'aider les Congolais à passer à travers la crise n'a pas suffi pour soulager leur souffrance. Des entrevues effectuées auprès des communautés les plus fragiles montrent que ces dernières ont développé un sentiment d'abandon en se considérant comme des laissés-pour-compte. En revanche, les dirigeants ont continué à faire valoir un discours plus optimiste. De telles perceptions opposées sont néfastes pour la paix sociale. Consacré à l'évaluation des programmes nationaux de gestion de crise, l'article se veut une contribution pertinente à la littérature sur la consolidation de la paix. En essayant de se démarquer des analyses dominantes sur la paix en Afrique, le papier examine la capacité d'un pays comme la RDC à préserver la paix dans un contexte de crise de l'ampleur de la Covid-19.

Mots-clés : Covid-19 - Défis- Initiatives - Perceptions - Paix sociale RDC

\section{Abstract}

This research discusses the effects of Covid-19 on the poorest population in the Democratic Republic of Congo. The restrictive measures taken by the Congolese authorities during the first phase of the pandemic to contain the crisis have created new problems. They have considerably affected the already worrying socio-economic conditions of the most vulnerable groups. With so few means available to the country, the government's willingness to help the Congolese get through the crisis was not enough to alleviate their suffering. Interviews conducted with the most fragile communities show that they have developed a feeling of abandonment by seeing themselves as left behind. In contrast, leaders continued to push for a more optimistic rhetoric. Such perceptions are harmful for social peace. Dedicated to the evaluation of national crisis management programs, the article is intended to be a relevant contribution to the literature on peacebuilding. Trying to distance itself from dominant analyzes on 
peace in Africa, the paper examines the ability of a country like the DRC to preserve peace in the context of a crisis of the magnitude of Covid-19.

Keywords: Covid-19 - Challenges-Initiatives - Perceptions - Social peace - DRC 


\section{Introduction}

La pandémie de la Covid-19 a entraîné des conséquences douloureuses à travers la planète. En avril 2021, soit un peu plus d'un an après le déclenchement de la crise, le bilan mondial s'élevait à 3 millions de morts (Radio-Canada, 2021). Des États-Unis à la France en passant par le Brésil, les grandes puissances et les puissances émergentes demeurent les plus touchées. Malgré les moyens colossaux à leur disposition, ces pays éprouvent les répercussions les plus importantes du Coronavirus. Que ce soient en termes des dommages sanitaires ou des tensions économiques et sociales qui en résultent (Chesnais, 2020). Bien qu'elle n'ait pas occasionné des drames humains de la même ampleur que dans le reste du monde, la Covid-19 en Afrique subsaharienne nécessite néanmoins d'être soumis à un examen critique. II est particulièrement important d'analyser les pays comme la République Démocratique du Congo (RDC) qui tentent difficilement de se redresser des décennies de guerre (De Villers, 2005). La pandémie est venue amplifier la situation économique déjà fragile sur le continent. Certes, la lente propagation du virus dans la région permet, à ce jour, à certaines économies de mieux résister (Banque mondiale, 2021) ; la perspective d'une première récession depuis 25 ans fait cependant craindre des lendemains incertains et un «impact considérable sur les ménages [les plus vulnérables] » (Calderon et al, 2020, p. 1).

Cet article tente de répondre à la question suivante : quels sont les effets de la Covid-19 sur les conditions de vie des populations les plus démunies de la République Démocratique du Congo ? Nous portons une attention particulière aux programmes nationaux mis en place par les autorités congolaises dans le but de contenir la propagation du virus. Dans la mesure où l'absence de politiques favorables au bien-être social est régulièrement décriée dans ce pays (Arquillière et al, 2015), examiner les mesures gouvernementales dans ce contexte particulier constitue un objet d'étude irréfutable. Pour cela, nous analysons le plan d'action de lutte contre le Coronavirus élaboré par le gouvernement congolais que nous avons observé sur le terrain en tout début de la crise. Nous avons également conduit des entrevues auprès de la population qui est la principale bénéficiaire de 
ces programmes. Tout en explorant les défis auxquels le gouvernement congolais a été confronté, le papier s'attache à savoir jusqu'à quel point ses initiatives ont affecté la paix sociale. Notre recherche se focalise sur ce qu'un pays en difficulté économique et qui s'efforce de consolider la paix est capable de réaliser en l'absence de l'aide internationale. II faut dire que dans les premières semaines de la pandémie, les principaux contributeurs aux programmes de consolidation de la paix manquaient à l'appel. Les dirigeants comme Emmanuel Macron se contentant à ce stade de simplement appeler à un G20 extraordinaire chargé d'organiser « une réponse internationale à grande échelle » (Élysée, 2020). De plus, la menace de récession qui pesait également sur les économies occidentales, n'augurait pas de jours meilleurs sur le terrain de la solidarité internationale (Gnimassoun et Tapsoba, 2020).

Notre étude s'inscrit dans le corpus de travaux consacrés à la consolidation de la paix en Afrique. Le constat est que la majorité de ces recherches se concentre généralement sur les thèmes dominants de construction de la paix, tels que le rétablissement de la paix, les opérations de l'ONU, l'aide financière, la reconstruction des institutions, etc. (Autesserre, 2011 ; Onyejekwe, 2005; Rakotonirina, 2007; Vircoulon, 2007; Maendeleo Rutakaza, 2014 ; Vircoulon, 2005). Bien souvent, ces thématiques suggèrent aux pays bénéficiaires le statut d'assistés sociaux. Ils n'interrogent que rarement les initiatives locales de renforcement de la paix. En initiant cette étude, nous avons voulu nous projeter dans un scénario où l'entraide internationale était indisponible. Cela nous a poussé à nous intéresser au sort des populations des pays en difficulté économique comme la RDC. Nous verrons que le gouvernement congolais disposait de ressources limitées pour faire face à la pandémie, que les mesures prises avec les moyens du bord ont fortement affecté les groupes de population en situation de précarité.

\section{Initiatives nationales et paix sociale}

Les pratiques nationales en matière de bien-être social constituent un objet d'étude pertinent, du fait de leur capacité d'être la réponse la plus rapide aux besoins immédiats de la population. Elles 
concernent avant tout la meilleure redistribution des ressources disponibles, qui garantit une société plus équitable (Rawls, 1987). En cas de situation d'urgence, ces pratiques peuvent se manifester par le recours à des fonds spéciaux alloués aux œuvres de solidarité. En RDC, ces opérations sont mises en œuvre à travers une série d'initiatives comme le Plan d'organisation de secours en cas de catastrophe, dit PLAN ORSEC (Gouvernement de la république, 2012). Ce document prévoit une réaction rapide et efficace lors de calamités naturelles, mais aussi une assistance appropriée aux personnes affectées. De plus, il définit, bien avant que le désastre se produise, la façon dont les processus de secours peuvent être améliorés. En effet, ces procédures sont perfectionnées à travers la mise en place d'un leadership efficace et l'affectation de moyens humains, matériels et financiers suffisants. Toutefois, ce document reste souvent muet quand on en a le plus besoin. À la place, c'est le ministère des Affaires sociales, Actions humanitaires et Solidarité nationale créé depuis la transition de 2003-2006 qui est souvent chargé de porter secours aux sinistrés. II bénéficie de l'appui du gouvernement mais peut aussi compter sur la générosité des partenaires extérieurs et des Congolais. S'il manque les moyens de bien mener sa politique, le Département reconnaît néanmoins la capacité de destruction du tissu social que peuvent avoir les événements imprévus : " ils peuvent entraîner une aggravation de la vulnérabilité des personnes victimes, mais aussi une augmentation imprévisible des besoins sociaux ", peut-on lire dans son plan de protection sociale élaboré en 2016 (Ministère des Affaires sociales, 2016, p. 55).

De leur côté, quand elles le peuvent, les institutions provinciales, dans le cadre de la décentralisation, recourent également à des politiques similaires pour répondre à certaines urgences. C'est le cas par exemple du gouvernement provincial du Nord-Kivu qui avait utilisé des fonds propres en 2010 pour venir en aide aux populations sinistrées de la localité de Kibirigya (Reliefweb, 2010). Victimes de glissement de terrain, 19 personnes avaient trouvé la mort, tandis que plus de 200 ménages avaient perdu leurs habitations. Les dirigeants provinciaux n'ont pas seulement fourni plusieurs tonnes de produits alimentaires et d'autres objets de 
première nécessité, ils ont également montré la voie. Par cette intervention, insuffisante, selon les propos du gouverneur de l'époque, Julien Paluku, ils entendaient inciter les organismes humanitaires internationaux à apporter une aide beaucoup plus conséquente.

Certes, ces initiatives restent insignifiantes et se limitent souvent à de simples intentions. Elles montrent toutefois qu'un gouvernement moins équipé est à même d'envisager des réponses aux catastrophes naturelles avant de compter sur l'aide extérieure. En revanche, la faiblesse de ces moyens mérite d'être examinée, à partir du moment où elle devient un facteur de frustration pour une partie des Congolais. On peut d'ailleurs le constater à chaque inondation provoquée par les pluies diluviennes dans les quartiers défavorisés. À chaque fois, la colère monte d'un cran, à l'image de cette habitante du quartier Ndanu qui s'exprimait en 2015: «ils nous tuent en nous affamant, maintenant ils ont décidé de nous exterminer avec l'eau » (Agence France Presse, 2015). Ces propos recueillis par un journaliste de l'Agence France Presse au lendemain d'une pluie meurtrière à Kinshasa témoignent de la fragilité des rapports entre les dirigeants congolais et la population. Ils montrent à quel point une détresse peut à tout moment ranimer le ressentiment des victimes déjà asphyxiées par un vécu quotidien ardu. Malgré la volonté des gouvernants d'apporter des solutions, une crise de grande envergure comme une forte pluie ou une pandémie expose considérablement le pays à des défis difficiles à gérer. Cette situation est encore plus compliquée pour un pays post-conflit, où les habitants considèrent régulièrement les dirigeants comme responsables de leur souffrance.$^{23}$ Avec une économie à bout de souffle détruite par les années de conflits armés, la RDC figure parmi les pays africains ayant la population la plus pauvre. Ce classement s'est d'ailleurs aggravé avec la crise sanitaire sous étude (Banque Mondiale, 2021). Avec de maigres ressources, le pays doit ainsi régulièrement faire face à des demandes qu'il a souvent du mal à satisfaire. Ces demandes sont d'autant plus pressantes que les autorités sont fréquemment sommées de justifier la moindre dépense « futile ». Un simple achat

${ }^{23} \mathrm{Ce}$ sentiment est revenu régulièrement parmi les personnes que nous avons interrogées. 
de véhicules de luxe pour le transport des élus, par exemple, ne manque pas de provoquer un scandale d'état (Radio Okapi, 2021).

Ainsi, porter attention aux initiatives nationales conduit directement à palper du doigt leur impact sur la vie des gens. Cela permet également de souligner la tension que ces mesures sont susceptibles de provoquer. De fait, cette approche est cruciale pour identifier les inégalités potentielles générées par les décisions publiques. Comme le rappelle Virginia Held (2006, p. 10-11), il s'agit de s'intéresser prioritairement aux besoins essentiels, à la survie et aux droits des groupes défavorisés. Intégrer la dimension de justice sociale dans les études sur la paix s'avère donc une nécessité dans un contexte de crise comme celui de la Covid-19. Dans la section suivante, nous présentons les contours de ce modèle d'analyse. Nous allons voir à quel point il peut nous aider à comprendre les initiatives prises par le gouvernement congolais pendant la première partie de la crise, ainsi que leurs effets potentiels sur la paix sociale.

\section{La justice sociale comme réponse inclusive et facteur de paix sociale}

Nous avons soutenu qu'une analyse des processus de paix intégrant la dimension de justice sociale était appropriée pour comprendre les initiatives nationales des pays en situation de postconflit et leur impact sur les populations vulnérables. II convient de noter qu'il existe plusieurs visions de justice sociale avec des objets différents. Une première catégorie d'analyses est celle qui met en avant les structures. Ses théoriciens insistent, à l'instar de John Rawls (1987), sur l'idée de justice comme " structure de base de la société ». Ils s'attardent sur la façon dont les « institutions sociales » les plus importantes de la société redistribuent les «droits et les devoirs fondamentaux ». L'idée centrale de cette vision est que dans toute société, la " structure » comporte différents " niveaux sociaux ». II y a les gens du haut de l'échelle et ceux du bas de l'échelle. Par conséquent, ces individus expérimenteront des "perspectives de vie » différentes selon la position sociale que leur confèrent lesdites institutions (Rawls, 1987, p. 34). Cette conception de la structure institutionnelle souligne la nécessité de construire des institutions 
capables de limiter les inégalités. Ce besoin est encore plus prégnant dans les contextes comme ceux de l'urgence sanitaire où les demandes explosent à tous les niveaux. II incite à concevoir des réponses appropriées et à les adapter à toutes les demandes conformément à la position qu'occupe chaque membre de la communauté.

La seconde catégorie d'analyses questionne les grandes études sur les performances économiques au sein des États. Elle se focalise sur les conditions existentielles des individus. Elle considère que l'intérêt des spécialistes sur les chiffres, la " croissance économique » ou le " développement » ne pourrait avoir de pertinence que si tous ces concepts avaient une incidence positive sur la vie des gens (Sen, 2010, p. 277-278). En effet, au cours de dernières années, les pays comme la RDC ont connu des performances économiques record. En 2013, par exemple, Kinshasa atteignait une croissance à deux chiffres. Le taux d'inflation passait de $511,2 \%$ à $0,3 \%$, soit « le plus bas niveau jamais atteint depuis 1970 ", s'est même félicité le premier ministre d'alors, Augustin Matata Ponyo (Kibangula, 2013). Cependant, ces bons chiffres ne se sont que rarement traduits dans le panier de la ménagère. Bien au contraire, le pouvoir d'achat des Congolais a continué de plonger. Se nourrir, se soigner ou scolariser ses enfants reste une lutte permanente que la pandémie de la Covid-19 est venue intensifier davantage.

Nous examinons la situation en RDC à la lumière du modèle de justice sociale élaboré par Mertens et Wilson (2012). Ce modèle est plus approprié car il concerne l'application de la justice sociale à la mise en œuvre des programmes. Ses deux auteurs le qualifient d'ailleurs d'approche transformatrice. II s'agit pour eux de mettre l'emphase sur son objectif, lequel vise à encourager les plans d'action qui apportent le changement au niveau social (Mertens et Wilson, 2012, p. 161). Le principal argument de ce paradigme de transformation se concentre sur les enjeux de "pouvoir " et de "luttes contre les inégalités". II fait par ailleurs la promotion des "droits de l'homme » et de la " justice sociale » (Mertens et Wilson 2012, p. 162). D'autre part, ce cadre d'analyse puise dans les idées de Kincheloe et McLaren (2005), pour lesquels, l'analyse sensible à la 
justice sociale devrait aller au-delà de questions de "pouvoir » et de " justice ». Les enjeux de " race », de statut social, d'appartenance sexuelle, de convictions idéologiques, de niveau d'éducation, de croyances religieuses et de culture, devraient interconnecter afin de bâtir un "système social » inclusif (Kincheloe et McLaren, 2005, p. 92). Pour Mertens et Wilson, quiconque fera preuve d'une telle prise de conscience lancera un signal fort en termes de reconnaissance des inégalités et se donnera les moyens de les corriger.

Appliquées aux initiatives publiques comme les mesures gouvernementales de lutte contre la Covid-19, ces analyses donnent une orientation claire à propos des répercussions potentielles sur les groupes défavorisés. Elles invitent à prendre en compte le fait que la " discrimination » et la répression sont des éléments toujours présents dans les processus de distribution des programmes. II est donc important d'être à l'écoute des communautés. Ce faisant, on parvient à remettre en question les mécanismes sociétaux en place, qui favorisent la pérennisation de la précarité au sein des communautés (Mertens et Wilson, 2012, p. 164). Cette orientation analytique est intéressante parce qu'elle cherche à démontrer le risque d'exclusion et de discrimination dans la mise en œuvre des initiatives destinées à soulager la population. Elle fournit une base de réflexion en attirant l'attention sur l'importance de prêter une oreille attentive aux bénéficiaires. C'est le seul moyen d'identifier les problèmes spécifiques et la diversité des profils au sein des communautés, ce qui permet une prise en charge plus équitable. Finalement, c'est en faisant la promotion de la justice sociale et des droits de la personne, qu'on peut parvenir à l'application des mesures qui permettent de mettre fin aux inégalités et de mettre tous les groupes à l'abri du danger.

En ce qui concerne la consolidation de la paix, l'approche axée sur la justice sociale offre l'avantage de concentrer la réflexion sur les politiques directes décidées localement. Elle donne par ailleurs la possibilité d'évaluer les défis auxquels les États sont confrontés dans la mise en œuvre des programmes, l'efficacité desdites mesures et leur effet sur la paix sociale. Nous allons voir dans la section qui suit la réponse du gouvernement congolais à la pandémie et les programmes décidés en faveur de la population. 
Compte tenu de l'immensité du territoire national de la RDC, notre enquête s'est limitée à Kinshasa. Effectuer une telle étude dans la capitale congolaise où siège des institutions, vous donne un accès facile aux sources officielles et aux documents importants. Nous avons opté pour une recherche exploratoire, c'est-à-dire, en partant de l'annonce des mesures officielles jusqu'à leur perception au sein de la population. Pour cela, nous avons conduit des entrevues semidirigées selon l'approche de Lorraine Savoie-Zajc (2010). L'avantage de cette forme d'entretiens est qu'elle implique une interaction verbale riche entre le chercheur et le répondant. Ce dernier partage son expérience vécue qui permet de dégager de façon conjointe la compréhension du phénomène étudié. De plus, avec cette technique de collecte de données, il est plus facile de s'ajuster selon le profil des personnes interrogées (Blais et Durand, 2010). Le questionnaire adressé à notre population-cible s'est articulé au tour de l'impact des mesures du gouvernement contre la Covid-19 sur son vécu quotidien, tandis que les représentants du gouvernement devaient nous apporter leur expertise sur lesdites initiatives. Tout en prenant en compte les problèmes des provinces régulièrement relayés par les médias kinois $^{24}$, notre étude s'est intéressée à la situation dans les quartiers défavorisés de Kinshasa. Nous nous sommes spécifiquement concentrés sur le quartier Mbanza Lemba, l'un des plus pauvres de la ville.

\section{Les mesures prises par le gouvernement congolais}

La première mesure est venue du président de la République lui-même. Dans un message solennel à la nation le 24 mars 2020, Félix Tshisekedi décrète l'état d'urgence sanitaire. II met ensuite en place une Task Force de lutte contre la Covid-19. À l'instar d'autres pays du monde, la fermeture des frontières est imposée pour éviter toute intrusion du virus sur le territoire national. Devant la panique qui s'empare de la planète, le gouvernement congolais va même plus loin que la France ou les États-Unis en interdisant tous les voyages de Kinshasa vers les provinces, et vice-versa. Déclaré foyer de la

\footnotetext{
${ }^{24}$ La radio onusienne Okapi couvre l'ensemble du territoire national et remonte chaque matin le vécu quotidien des Congolais du pays profond. 
pandémie, la capitale congolaise doit être strictement confinée. Fermer un pays où la majorité de la population survit du jour au jour crée cependant des problèmes supplémentaires. À l'article 6 de son Ordonnance présidentielle, le chef de l'État n'oublie d'ailleurs pas de le mentionner :

Le Gouvernement de la République met en place des modes appropriés d'approvisionnement des villes en denrées alimentaires pour prévenir toute rupture de stock afin de mettre la nation à l'abri de toutes conséquences désastreuses sur le plan de la sécurité alimentaire et des troubles éventuels à l'ordre public. (Extrait de l'Ordonnance présidentielle du 24 mars 2020, article 6)

La fermeture des frontières intérieures et les marchés risquait de transformer la crise sanitaire en conflit social. L'économie congolaise étant extravertie et reposant essentiellement sur l'exportation des ressources minières et forestières, il était impératif d'intervenir sur le terrain social et de trouver une solution palliative. Cela était nécessaire pour prévenir une crise de la faim, nous a expliqué Taylor Katayi Tshimanga. Conseiller en charge des questions économiques auprès du ministère de l'Économie, nous lui avons demandé quel a été le plan du gouvernement pour aider les ménages les plus vulnérables :

Intervenir veut dire avoir déjà les moyens pour subvenir aux besoins de ceux qui vous regardent. Ces moyens n'étaient pas disponibles, vous connaissez notre budget et vous savez qu'il fallait les mobiliser. Mais, quand même, on a réfléchi et une grande action a été prise. Celle-là a porté sur l'annulation de la TVA [Taxe sur la valeur ajoutée]. C'est la plus grande mesure que nous avons prise. On a annulé la TVA sur la consommation de quelques biens de première nécessité. Cela a fait que les produits sur le marché ont gardé un certain frein par rapport à l'inflation des prix à laquelle on pouvait 
s'attendre en période normal ou en période d'inaction du gouvernement, sur ce point-là en particulier. En annulant la TVA, on a ajouté le contrôle économique pendant cette période très dure.

Ce contrôle a été très effectif auprès des importateurs des produits de consommation. Parce que nous savons que les opérateurs économiques ici dans le pays sont assez véreux. Donc, c'était pour eux une opportunité pour hausser les prix et faire souffrir les consommateurs. Les pauvres consommateurs qui devaient gérer la maladie, le manque d'argent et de travail et l'incapacité à sortir de chez eux pour aller chercher de quoi manger - alors qu'ici chez nous, nous parlons souvent de l' " article 15 », qui veut dire, qu'il faut se débrouiller pour manger. Voyez-vous? Ce sont des difficultés qu'on ramassait comme ça à la pelle et pour lesquelles il fallait qu'on se batte.

Combattre l'augmentation des prix était l'un des principaux champs d'action du gouvernement alors que la banque centrale du Congo $(B C C)$ tirait déjà la sonnette d'alarme. Les données du comité de politique monétaire de la BCC consultées situaient en effet l'inflation à plus de $9 \%$, soit, 2 points de plus qu'au début de la pandémie, avec la possibilité d'atteindre 15\% à la fin de l'année 2020, si des mesures adéquates n'étaient pas prises (Radio Okapi, 2020). On notera ici l'explosion de l'inflation (de $0,3 \%$, à $9 \%$ ) en 7 ans. Pour les autorités congolaises, il fallait passer un nouveau cap, à savoir, ravitailler les Congolais en produits alimentaires. Taylor Katayi Tshimanga explique :

C'est vrai qu'ils [les opérateurs économiques] avaient peur parce que les importations ne venaient pas ou alors elles ne venaient pas au bon rythme [à cause de la fermeture des frontières]. Mais nous avons travaillé sur la gestion des stocks. Nous nous sommes renseignés sur tout ce qu'il y avait en stock et puis par rapport au besoin normal du marché, 
nous nous sommes battus pour les forcer à ne pas retenir les stocks en magasin, ce qui pouvait créer une rareté et avoir des conséquences néfastes sur les prix de biens de consommation intérieure [...]. Avec l'aide de la présidence, nous avons recouru aux actions d'approvisionnement dans les sites de consommation.

On a créé des structures instantanées qui avaient pour rôle essentiel de rentrer à l'intérieur du pays dans les différents centres de production qui sont enfuis dans nos régions, de récupérer les produits et de les ramener vers les centres de consommation. [...] On a importé le riz et les maniocs. Et quand ces produits arrivent ici [à Kinshasa], si un sac de manioc, coûtait 60 dollars USD, les sacs qui viennent des ONG que nous soutenons vont coûter 40 ou 30 dollars USD. Ça influence tous ceux qui distribuent sur le marché. [...]. Et pour les Congolais, c'était comme de la magie. Ils se sont dit: « mais qu'est-ce que le gouvernement a fait pour que pendant cette période difficile tant redoutée et où le monde entier pleure les prix des biens de première nécessité soient en train de baisser? »

Cette explication suggère que le gouvernement congolais a paré au plus pressé en suivant la dynamique mondiale caractérisée par la fermeture des frontières nationales. Cependant, l'interdiction des déplacements interprovinciaux combinée à la fermeture des principaux marchés a révélé un grand risque de crise de la faim. Autrement dit, ces décisions ont exposé le pays à une possible explosion sociale. En décidant de soutenir les entreprises du secteur alimentaire tout en les concurrençant au niveau des prix, le gouvernement congolais voulait montrer qu'il était prêt à tout pour éviter les conséquences désastreuses sur le plan social. Le président congolais Félix Tshisekedi l'a lui-même reconnu 3 mois après avoir instauré un état d'urgence sanitaire (Agence congolaise de presse, 2020). II est revenu devant la nation à la fin du mois de juin 2020 pour 
lancer un nouveau programme d'urgence. Évalué à 2, 610 milliards USD, ce nouveau plan visait non seulement à renforcer la riposte contre la Covid-19, mais aussi à préserver la vie des Congolais, selon le chef de l'État.

\section{Une population compréhensive mais exposée à la précarité}

Pour le gouvernement congolais, le défi était de donner un sens à la notion de " préserver la vie des Congolais ". Avec plus de $77 \%$ de Congolais vivant de l'économie informelle et plus de $90 \%$ de ménages dépendant de ce seul secteur (Malula, 2020), il était impossible de recourir à des mesures liées à l'emploi. Certains pays mieux lotis économiquement n'ont pas hésité d'adopter une politique très généreuse en versant de l'argent aux travailleurs pour les inciter à rester à la maison. C'est le cas par exemple du Canada avec sa Prestation canadienne d'urgence (Gouvernement du Canada, 2020). Un luxe que la RDC ne pouvait pas se permettre. Aider les importateurs en baissant les charges fiscales et s'improviser dans la vente des denrées alimentaires semblait être les seuls leviers à la portée des autorités congolaises. Mais si ces mesures permettent de montrer l'image d'un gouvernement soucieux du sort de ses citoyens, dans les faits, soulager la souffrance des 100 millions de Congolais est compliqué à matérialiser avec si peu de moyens. La mégalopole Kinshasa illustre à elle seule ce défi. Avec plus de 10 millions d'habitants, la capitale congolaise concentre l'essentiel de l'attention des autorités. Pourtant, même ici, les initiatives prises par le gouvernement n'ont pas suffi pour mettre tout le monde à l'abri d'une crise alimentaire.

Pour vérifier cette réalité, nous nous sommes rendus au quartier Mbanza Lemba, l'un des plus pauvres de la ville. Située au pied de l'Université de Kinshasa, cette cité symbolise le paradoxe que vivent une partie de Kinois. Ils se trouvent à la fois si proche du haut lieu du savoir en RDC et si loin du centre d'affaires. Cet éloignement du centre-ville les prive non seulement d'électricité et d'eau potable, il les tient également à l'écart de la précieuse attention des dirigeants. Pour notre étude, ce quartier constituait le cadre idéal pour collecter les données et ainsi procéder à l'évaluation de la gestion de la crise 
de la Covid-19 par les autorités congolaises. Nous y avons donc réalisé une trentaine d'entrevues en suivant le modèle décrite précédemment. À noter que l'ensemble des répondants nous a raconté quasiment les mêmes conditions de vie difficiles, ce qui rendait leurs propos redondants. Afin de simplifier l'exploitation de données, nous avons fait le tri et constitué un corpus d'analyse de quinze entrevues. Avec cinq femmes, cinq hommes et cinq jeunes majeurs tout sexe confondu, nous avons formé ces catégories représentatives du profil général des habitants de la banlieue kinoise. Âgés de 25 à 60 ans, les répondants partagent en commun les inquiétudes relatives à la perte de leur activité quotidienne à cause de la fermeture des principaux marchés. Vendeurs à la sauvette ou sur les étalages de fortune, ils ont souvent été obligés de changer constamment de métier selon les circonstances. Pour ces débrouillards tout terrain, il fallait s'accrocher à n'importe quoi, pourvu que cela leur permette de rapporter de quoi manger à la maison. Nous leur avons demandé comment ils ont vécu le confinement et s'ils ont reçu suffisamment de soutien de la part du gouvernement. Tout en se montrant compréhensifs des mesures restrictives et des efforts consentis par les autorités pour contenir la crise, dans l'ensemble, les répondants font part de conditions de vie « volatiles »:

J'étais vendeuse de friperie dans le grand marché mais pendant le confinement, j'ai commencé à vendre du riz et du haricot que je proposais aux passants sur mon avenue. C'était compliqué pour ma famille de vivre avec ça, mais on n'avait pas de choix. Certainement, les riches et surtout les politiciens, avaient les moyens suffisants pour faire des provisions. Mais nous, nous étions totalement abandonnés. Avec cette pandémie, j'ai vraiment expérimenté l'injustice sur cette terre des hommes.

À l'instar de Monique, 60 ans, le sentiment de voir les inégalités augmenter en République Démocratique du Congo à cause 
de la pandémie du Coronavirus est répandu au quartier Mbanza Lemba. Aimé, 27 ans, partage cette inquiétude en accusant l'État de «nous avoir abandonnés »:

La période de confinement était vraiment pénible. Nous avons souffert avec nos enfants, étant donné que je ne vendais plus. Pour survivre, j'étais obligé de faire recours aux membres de ma famille. Pour moi, c'était une catastrophe. Mais eux [les dirigeants], surtout en politique, ils gagnent beaucoup d'argent. Ils ont des comptes en banque. Pendant que nous nous sommes abandonnés à nous débrouiller tout seuls.

Asphyxiés par le confinement, la plupart de répondants étaient aussi soumis au diktat des devises étrangères qui font la loi sur l'économie congolaise. Résultat, certains, comme Vanella, 26 ans, ont vu leur fonds de commerce devenir totalement inadapté à la réalité du marché :

Je suis vendeur de poissons, mais le confinement m'a ramenée à ma première activité, c'est-à-dire, à la vente des légumes. Tout cela parce que mon capital avait chuté à cause du dollar américain qui avait pris de la valeur sur le franc congolais. Nous ici, nous faisons tout en Franc congolais.

Pour Stanis, 55 ans, la faute incombe au gouvernement et sa passivité :

Ce qui m'a rendu dans cet état, c'est l'incapacité de nos dirigeants à stabiliser le taux de change. Je vendais les boudins dans une station, mais à l'arrivée du 
confinement, j'ai commencé à coiffer les cheveux des enfants dans mon quartier, simplement parce que mes frais de démarrage étaient partis en fumée avec vos affaires de taux de change. C'est ça le Congo et moi je n'ai jamais compté sur eux. [...]. Ils vivaient bien, mais nous nous étions en train de souffrir. Nous étions délaissés. Je regrette le désordre de notre gouvernement qui n'a pas su nous prendre en charge.

\section{Un gouvernement enthousiaste, une population inquiète}

Pour le gouvernement, en revanche, le compte était bon. Le fait d'avoir accordé des "facilités fiscales" aux opérateurs économiques a permis de limiter la casse. De plus, les dirigeants congolais se sont improvisés dans l'achat et la revente de denrées alimentaires, ce qui a permis, selon eux, d'approvisionner les grandes villes. Ces réalisations auraient même réjoui les Congolais, s'est enflammé un représentant du ministère de l'Économie. Cependant, la perception des principaux bénéficiaires reste très éloignée de l'enthousiasme ressenti au sommet de l'État. Ce constat est partagé par les organisations de la société civile. Jonas Tshombela dirige l'une d'elles :

[...] Les inégalités se sont amplifiées davantage avec le Coronavirus et la gestion de cette pandémie par le gouvernement. Le plan de redressement de l'économie et l'accompagnement des entreprises ont créé un environnement hostile au développement et ont accentué les inégalités. Aujourd'hui, l'État fait payer deux fois l'eau et l'électricité alors qu'on avait dit que c'était gratuit pendant la période de confinement. Le gouvernement n'a pas bien protégé la population. 
En reprenant le recouvrement des taxes et des factures, les autorités congolaises ont, selon la société civile, donné l'impression d'être insensibles à la situation difficile à laquelle est confrontée la population. Le gouvernement avait en effet besoin de recommencer à renflouer ses caisses y compris en sollicitant la poche des plus démunis. Pour le coordonnateur de l'Union pour la défense des consommateurs du Congo, Chef Tshipamba, cela montre que toute la planification de gestion de crise n'a fait l'objet d'aucun suivi pour garantir un bien-être social à tous. Elle aurait même, selon lui, creusé un peu plus les inégalités. Nous avons demandé à cet autre membre de la société civile si cette situation risquait de mettre en péril la paix sociale dans ce pays :

Les décisions prises n'étaient pas objectivement encadrées. La paix sociale était fragilisée dans la mesure où pendant le confinement nous ne travaillons pas mais nous devons nouer les deux bouts du mois. Pendant le confinement, les enfants ont consommé plus qu'ils ne le font lorsqu'ils partent à l'école. [...]. On avait l'impression que la Task force mise en place par le chef de l'État ne se concentrait que sur les décisions rendues publiques au niveau de la présidence, tandis qu'au niveau sectoriel, il n'y avait aucun suivi. Le gouvernement était un peu distrait et avait négligé le peuple.

Pendant le confinement, le prix du pain a augmenté, le gouvernement n'a rien dit, au sortir du confinement le ministre des Télécommunications nous a créé une taxe sur les appareils [téléphoniques]. Tout ceci ne peut que susciter des tensions. 
La différence des perceptions sur le bilan à mi-parcours de la gestion de la Covid-19 en République Démocratique du Congo semble évidente. D'un côté, les autorités se montrent plus optimistes en affirmant avoir fait l'essentiel. De l'autre, les mouvements associatifs et les Congolais les plus démunis partagent un sentiment de compréhension à l'égard des efforts consentis par les autorités. Ils expriment toutefois leur crainte de ne pas pouvoir se nourrir décemment à cause de difficultés persistantes. II est certes difficile pour un pays dont le budget atteint à peine 10 milliards USD ${ }^{15}$ de faire des miracles devant une situation aussi brusque que la Covid-19. Néanmoins, il était important d'examiner la capacité du gouvernement congolais à se soucier du sort des communautés les plus exposées aux impacts de la pandémie. Le meilleur moyen de s'en préoccuper aurait consisté, selon Virginia Held (2006, p. 3-10) ou David Miller (1999, p. 217-220), de s'interroger avant toute chose quant aux conditions existentielles des êtres pour lesquels nous avons la responsabilité et de tenter d'y apporter des solutions.

Les communautés du quartier Mbanza Lemba et leur débrouillardise quotidienne représentent le prototype de divers groupes les plus vulnérables qui vivent à Kinshasa mais aussi dans les 25 autres provinces congolaises. II s'agit de gens dépourvus d'emplois, qui survivent dans l'économie informelle peu confortable et vivent en permanence avec le sentiment d'être abandonnés. C'est ce qu'on peut souvent entendre dans leurs témoignages :

Chacun vivait de sa manière. Nous la basse classe, nous nous sommes sentis marginalisés, parce qu'ils n'ont eu aucun plan pour aider même les plus démunis. Les autorités n'ont pas pensé à nous et la pandémie n'a fait que renforcer les inégalités déjà présentes dans notre pays.

\footnotetext{
${ }^{15}$ La loi des finances 2020 prévoyait un budget en recettes et en dépenses à hauteur de 18, 545, 2 milliards de franc congolais, soit environs 10 milliards USD.
} 
Réputée être un scandale géologique, la République Démocratique du Congo dispose de ressources naturelles suffisantes pour faire de sa population l'une des plus heureuses au monde. Mais la mauvaise gouvernance, le pillage des richesses et les guerres à répétition ont longtemps miné la perspective de prospérité pour ce géant de l'Afrique centrale (Pourtier, 2002). Par conséquent, dès que le pays est confronté à une crise de la brutalité de la Covid-19, il se trouve totalement désarmé pour répondre efficacement aux besoins de sa population la plus exposée. Dès lors, le peu de mesures que peut initier le gouvernement comme l'approvisionnement en denrées alimentaires ou la maîtrise de l'inflation sur le marché apparaissent souvent comme une tempête dans un verre d'eau. Car les principaux bénéficiaires n'en ressentent que trop peu d'effet. Cette absence apparente de solution pourrait contribuer à creuser un peu plus le décalage entre les dirigeants et la population. Elle risque de renforcer le sentiment d'abandon parmi les groupes les plus démunis.

\section{Conclusion}

Les effets de la Covid-19 sur les conditions de vie des populations vulnérables de la République Démocratique du Congo sont sans équivoque. Non seulement la plupart des Congolais ont perdu leur maigre source de revenu, ils ont surtout développé le sentiment d'être des laissés-pour-compte par les dirigeants. La décision prise par les autorités de fermer les marchés était plus que nécessaire pour éviter la propagation de la maladie. En même temps, elle a condamné une population qui vit au taux du jour et de la débrouille. Vendeurs à la sauvette ou restaurateurs de fortune, tous ont été contraints de changer constamment de métier. Dans la majorité des cas, ces acrobaties n'étaient pas suffisantes pour nouer les deux bouts du mois. Sans alternative adéquate et soumis à rude épreuve, ces Congolais n'ont pas eu d'autres choix que de trouver un responsable à leur souffrance. Ils n'hésitent pas à pointer du doigt la classe dirigeante, la seule à disposer de comptes en banques, soulignent-ils, et la seule capable de s'approvisionner pendant ces dures mesures restrictives. De telles perceptions renvoient l'image également redoutée par John Rawls, celle d'une structure sociale désagrégée. Elles donnent à penser que la RDC est une société à 
deux échelles : une, composée des gens d'en haut, qui peuvent se protéger plus aisément des calamités naturelles et une autre occupée par les gens d'en bas abandonnés à leur triste sort. Dans la perspective de Mertens et Wilson, ces perceptions illustreraient l'absence d'institutions capables d'adresser les programmes qui prennent en compte les besoins des membres les plus vulnérables de la société.

En s'intéressant aux pratiques nationales dans une période de crise, cette étude a permis de soulever les questions souvent peu abordées dans la littérature sur la paix. Sans l'intervention internationale, on se rend compte de difficultés auxquelles les pays comme la RDC peuvent être confrontés. S'ils ont la volonté de venir en aide à leurs populations, la faiblesse de moyens à leur disposition ne les aide pas à atteindre cet objectif. De plus, les mesures drastiques que les dirigeants finissent par adopter semblent souvent inadaptées, causent plus de mal et renforcent les conditions déjà préoccupantes des populations. Sans ressources adéquates et contraints d'agir contre une pandémie dont les conséquences pouvaient être encore plus graves, les dirigeants congolais n'ont eux aussi pas eu tellement de choix. Ils se sont contentés d'un service minimum présenté comme une victoire aux Congolais. La réalité du terrain est cependant différente. Les témoignages des habitants de Mbanza Lemba montrent que le fossé déjà profond entre les gouvernants et la population a continué de se creuser. Certes, la RDC n'a pas les moyens de la France ou du Canada pour prendre en charge tous ses citoyens. Des efforts devraient néanmoins être consentis pour parvenir, à moyen et long terme, à une distribution équitable des programmes publics. C'est du moins ce que la pandémie de la Covid-19 et son lot de dégâts permettent de rappeler. Et c'est fondamental pour préserver la paix sociale.

\section{Bibliographie}

Agence congolaise de presse. (2020, 19 juin). Le Président Félix Tshisekedi lance le Programme multisectoriel d'urgence d'atténuation des impacts de la COVID-19 en RDC. FAAPA. 
http://www.faapa.info/blog/le-president-felix-tshisekedilance-le-programme-multisectoriel-durgence-dattenuationdes-impacts-de-la-covid-19-en-rdc/

Agence France Presse. (2015, 9 décembre). À Ndanu, quartier inondé de Kinshasa, la colère gronde contre les autorités. L'express. https://www.lexpress.fr/actualites/1/styles/andanu-quartier-inonde-de-kinshasa-la-colere-gronde-contreles-autorites_1744228.html

Arquillière, M., Richard, D. et Peigner, C. (2015). L'instabilité en République Démocratique du Congo : entre conflit ouvert, corruption et ingérence économique. Irénée.net. https://www.irenees.net/bdf_fiche-analyse-1036_fr.html

Autesserre, S. (2011). Construire la paix : conceptions collectives de son établissement, de son maintien et de sa consolidation. Critique internationale, (51), 153-167.

Banque mondiale. (2001, 31 mars). Au cœur de la récession, l'Afrique subsaharienne entrevoit la reprise économique. https://www.banquemondiale.org/fr/news/pressrelease/2021/03/31/amid-recession-sub-saharan-africapoised-for-recovery

Banque Mondiale. (2021, 28 avril). République Démocratique du Congo - Vue d'ensemble. https://www.banquemondiale.org/fr/country/drc/overview

Blais, A. et Durand, C. (2010). Le sondage. Dans Benoît Gauthier (dir.), Recherche sociale :de la problématique à la collecte de données. Presses de l'Université du Québec.

Calderon, C., Kambou, G., Djiofack, C. Z., Kubota, M., Korman, V. \& Cantu Canales, C. (2020). Africa's Pulse, 21.

https://documents1.worldbank.org/curated/en/95098158646 2612240/pdf/Executive-Summary.pdf

Chesnais, F. (2020). L'état de l'économie mondiale au début de la grande récession Covid-19: repères historiques, analyses et illustrations. À l'encontre. http://alencontre.org/laune/letatde-leconomie-mondiale-au-debut-de-la-grande-recessioncovid-19-reperes-historiques-analyses-et-illustrations.html de Villers, G. (2005). La guerre dans les évolutions du CongoKinshasa. Afrique contemporaine, (215), 47-70. 
Élysée. (2020). Déclaration finale du Sommet extraordinaire des chefs d'État et de gouvernement du G20 consacré au COVID-19. Élysée.fr. https://www.elysee.fr/emmanuelmacron/2020/03/26/declaration-finale-du-sommetextraordinaire-des-chefs-detat-et-de-gouvernement-du-g20consacre-au-covid-19

Gnimassoun, B. et Tapsoba, J. (2020, 7 juin). La pandémie de Covid-19, une occasion historique de réinventer le développement de l'Afrique. The Conversation. https://theconversation.com/la-pandemie-de-covid-19-uneoccasion-historique-de-reinventer-le-developpement-delafrique-139697

Gouvernement de la république, RDC. (2012). Plan d'organisation de secours en cas de catastrophe. PLAN ORSEC. Ifrc.org. https://www.ifrc.org/docs/IDRL/RDC\%20Plan.pdf Gouvernement du Canada. (2020). Prestation canadienne d'urgence. https://www.canada.ca/fr/services/prestations/ae/pcuscapplication.html

Held, V. (2006). The ethics of care: personal, political, and global. Oxford University Press.

Kibangula, T. (2013, 7 juin). Matata Ponyo : la RD Congo, un pays émergeant en 2030. Jeune Afrique. https://www.jeuneafrique.com/19148/economie/matataponyo-la-rd-congo-pays-mergent-en-2030/

Kincheloe, J-L. \& McLaren, P. (2005). Rethinking critical theory and qualitative research. In $\mathrm{N}$.

Denzin \& Y. S. Lincoln (Eds.), The SAGE handbook of qualitative research (3rd ed., p. 303-342). Sage.

Maendeleo Rutakaza, R. (2014). Le rétablissement et la consolidation de la paix en République démocratique du Congo de 1990 à 2008: le rôle des acteurs internationaux. L'Harmattan.

Malula, J. (2020, 20 avril). Covid-19 : l'impossible confinement de Kinshasa. Lepoint.fr.

https://www.lepoint.fr/afrique/covid-19-l-impossibleconfinement-de-kinshasa-20-04-2020-2372107 3826.php

Mertens, D-M. et Wilson, A-T. (2012). Program evaluation theory and practice: A comprehensive guide. Guilford Press. 
Miller, D. (1999). Principles of social justice. Harvard University Press.

Ministère des Affaires sociales - RDC. (2016). Politique nationale de protection sociale (volume1)

https://www.socialprotection.org/gimi/gess/RessourcePDF.action;jses sionid=MEDxhSg1Ys6p7MQliPIzbPWbUKIIEwrQr67e5QO OwqRz8HV-bGs!1750948109?id=55753

Onyejekwe, C. (2005). Les femmes, la guerre, la consolidation de la paix et la reconstruction. Revue internationale des sciences sociales, (184), 301-307.

Organisation International du Travail. (2020, 24 mars). Proclamation de l'état d'urgence sanitaire pour faire face à l'épidémie de Covid-19.

http://www.oit.org/dyn/natlex/natlex4.detail?p_lang=fr\&p_isn $=110918 \& p \_$country $=$COD\&p_classification $=01.08$

Pourtier, R. (2002). Le Congo (RDC) entre guerre et pillage (The Congo (DRC) amid war and plunder). Bulletin de l'Association de géographes français, 251-263.

Radio Canada. (2021). Évolution de la Covid-19 en temps réel. https://ici.radio-canada.ca/info/2020/coronavirus-covid-19pandemie-cas-carte-maladie-symptomes-propagation/

Radio Okapi. (2021, 21 juin). Africa News : « députés : 500 véhicules de la discorde ».

https://www.radiookapi.net/2021/06/21/actualite/revue-depresse/africa-news-deputes-500-vehicules-de-la-discorde

Radio Okapi. (2020, 18 juin). Banque centrale du Congo : la pandémie du Coronavirus ravage l'économie de la RDC. https://www.radiookapi.net/2020/06/18/actualite/economie/la -pandemie-du-coronavirus-ravage-leconomie-de-la-rdc-bcc

Rakotonirina, H. (2007). Compte rendu de [Faire la paix : concepts et pratiques de la consolidation de la paix Sous la dir. d'Yvan Conoir et Gérard Verna, Québec, Presses de L'Université Laval, 2005, 789 p.] Politique et Sociétés, 26(1), 174-180.

Rawls, J. (1987). La théorie de la justice. Paris: Seuil. Radio Okapi. (2010, 24 mai). RDC, Goma : une assistance du gouvernement provincial aux sinistrés de Kibirigya. Reliefweb. https://reliefweb.int/report/democratic-republic- 
congo/rdc-goma-une-assistance-du-gouvernementprovincial-aux-sinistr\%C3\%A9s-de

Savoie-Zajc, L. (2010). L'entrevue semi-dirigée. Dans B. Gauthier (dir.), Recherche sociale : de la problématique à la collecte de données. Presses de l'Université du Québec.

Sen, A. (2010). L'idée de justice. Paris : Flammarion.

Vircoulon, T. (2005). Ambiguïtés de l'intervention internationale en République démocratique du Congo. Politique africaine, (98), 79-95.

Vircoulon, T. (2007). L'Etat internationalisé : Nouvelle figure de la mondialisation en Afrique. Études, (406), 9-20. 